\title{
Fiber-Bragg-grating vibration sensor with temperature stability using wavelength-variable incoherent light source
}

\author{
Nobuaki Takahashi*, Weerapong Thongnum ${ }^{\dagger}$ and Sumio Takahashi ${ }^{\ddagger}$ \\ National Defense Academy
}

(Received 2 July 2002, Accepted for publication 17 July 2002)

Keywords: Vibration sensing, Optical fiber sensor, Fiber Bragg grating, Temperature stabilization, Incoherent light PACS number: 43.38.Zp, 43.40.Yq

\section{Introduction}

When a vibration is applied to a fiber Bragg grating (FBG), the FBG modulates intensity of narrow-spectrum incident light that has a wavelength tuned to the slope of the FBG reflection or transmission spectrum curve. Since the modulation of the optical intensity is in proportion to the applied vibration [1-3], the detection of the modulated light provides us with direct observation of the vibration. The sensitivity of the sensor is proportional to the slope of the spectrum curve at the operation wavelength. Although the principle is successfully applied to the vibration detection of a solid in the environment where the temperature change is small [4], it is known that large temperature change causes the sensor sensitivity to vary considerably because the Bragg reflection wavelength is shifted by not only applied strain but also temperature change of an FBG [1]. It is therefore generally necessary to stabilize the sensor against temperature change that may make the sensor operation unstable. In this paper we propose a new method to thermally stabilize an FBG vibration sensor in which the wavelength of the optical source follows the shift of the Bragg reflection wavelength of the sensing FBG. In the experiment, an external vibration is applied to the lead fiber of the sensing FBG and the vibration travels to the FBG along the fiber in order to avoid the influence of the possible vibration mode change of the vibrator.

\section{Principle}

An FBG is a special optical fiber that has periodical variation in index of refraction of the fiber core and reflects only the incident light that has a wavelength equal to the Bragg reflection wavelength of an FBG given by

$$
\lambda_{\mathrm{B}}=2 n_{0} \Lambda,
$$

where $n_{0}$ is the effective index of refraction of the core and $\Lambda$ is the pitch of the grating. As mentioned in the previous section, a vibration propagates along the lead fiber and varies both the refractive index and grating pitch when it reaches the FBG, which in turn results in a change of the Bragg reflection wavelength. The vibration then shifts the reflection spectrum curve periodically along the wavelength axis, e.g., from the solid to broken line in Fig. 1. For the sensor operation, we use

\footnotetext{
*e-mail: tak@nda.ac.jp

†e-mail: g40054@nda.ac.jp

‡e-mail: takahasi@nda.ac.jp
}

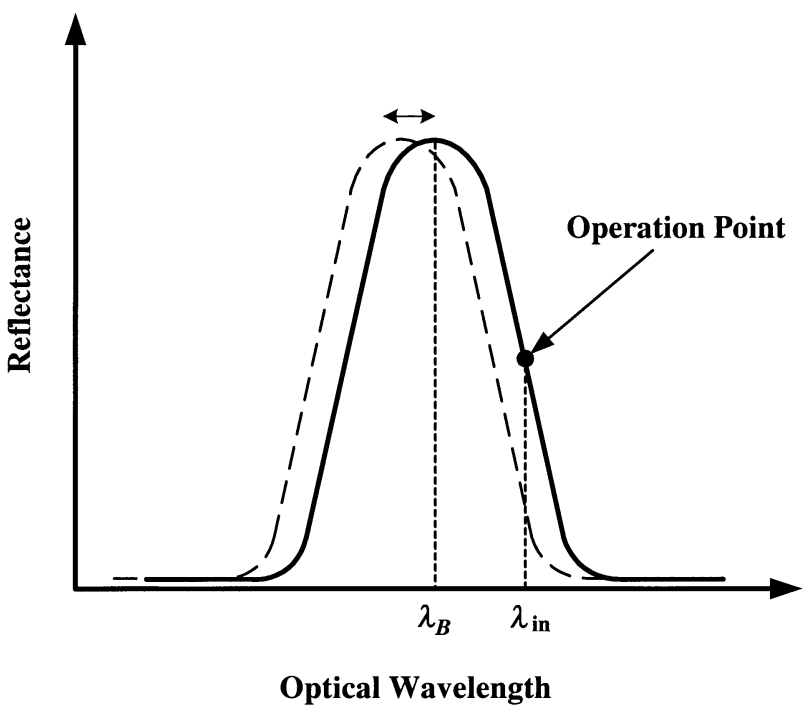

Fig. 1 Typical wavelength dependence of FBG reflectance and operation wavelength.

narrow-spectrum light as an optical source and tune the wavelength of the light to the slope of the reflection spectrum curve, for example, $\lambda_{\text {in }}$ in the figure. On reflecting back from the FBG, the light is modulated in intensity by the vibration. In thermally stabilizing the sensor, we have constructed an optical source for the sensor as shown in Fig. 2. The source consists of a broad-spectrum light source, which is an ASE (amplified spontaneous emission) output of an erbium-doped fiber amplifier (EDFA), an optical circulator and an FBG, which we call FBG1 from now on. This source outputs narrow-spectrum light of wavelength equal to the Bragg reflection wavelength of FBG1, which is located at the slope of the reflection spectrum curve of the sensing FBG, called FBG2. If the Bragg reflection wavelengths of the two FBGs have the same temperature dependence, the FBG2 reflection spectrum curve and source wavelength move together with changing temperature and the operation point remains unchanged on the spectrum curve regardless temperature change in the environment, i.e., the slope at the operation point is kept constant and the sensitivity of the sensor is thermally stabilized. 


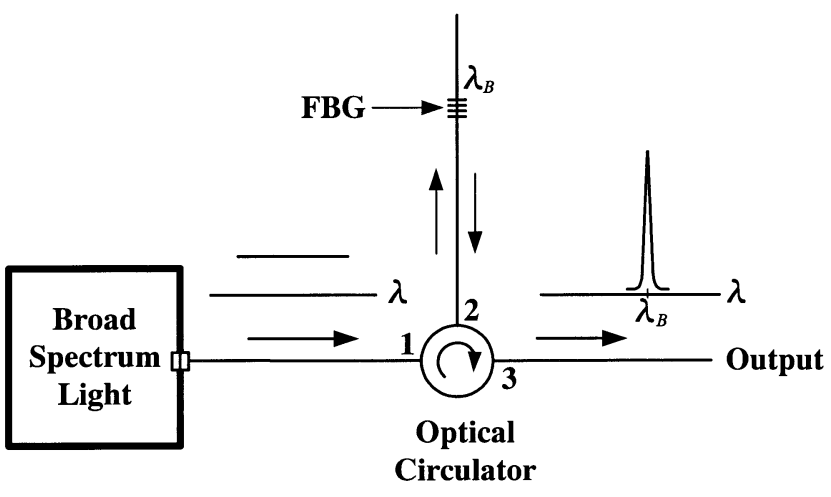

Fig. 2 Optical source for FBG vibration sensor.

\section{Experiment}

Figure 3 shows the experimental setup for the FBG vibration sensor that is thermally stabilized as described in the section 2. As mentioned above, two FBGs are used: One is for the optical source (FBG1) and another for sensing vibration (FBG2). The temperature dependence of the FBGs Bragg reflection wavelengths is shown in Fig. 4. One can see that the Bragg reflection wavelengths of the FBGs vary linearly with changing the temperature and the slopes of the FBG1 and FBG2 curves are practically same over the temperature range from -65 to $0^{\circ} \mathrm{C}$. If temperatures of the two FBGs are kept same, therefore, it is expected that the sensor will be stabilized in a temperature-varying environment. The vibrator used in the experiment is a bar-shaped PZT vibrator: The dimensions are $10 \mathrm{~mm}$ in width, $10 \mathrm{~mm}$ in thickness and $88 \mathrm{~mm}$ in length. During the measurements, the vibrator is driven at the resonance frequency, $19.040 \mathrm{kHz}$. The lead fiber of FBG2 is glued on the side surface of the vibrator in the middle along the length direction. The output light from the circulator 1 is amplified by an optical amplifier (EDFA) to obtain the power enough for operating the sensor and filtered out by an optical band-pass filter to eliminate undesirable wavelength components of the light. The light is then incident on FBG2 after passing through the circulator 2. Being intensity-modulated at FBG2, the light passes through the circulator 2 again and is

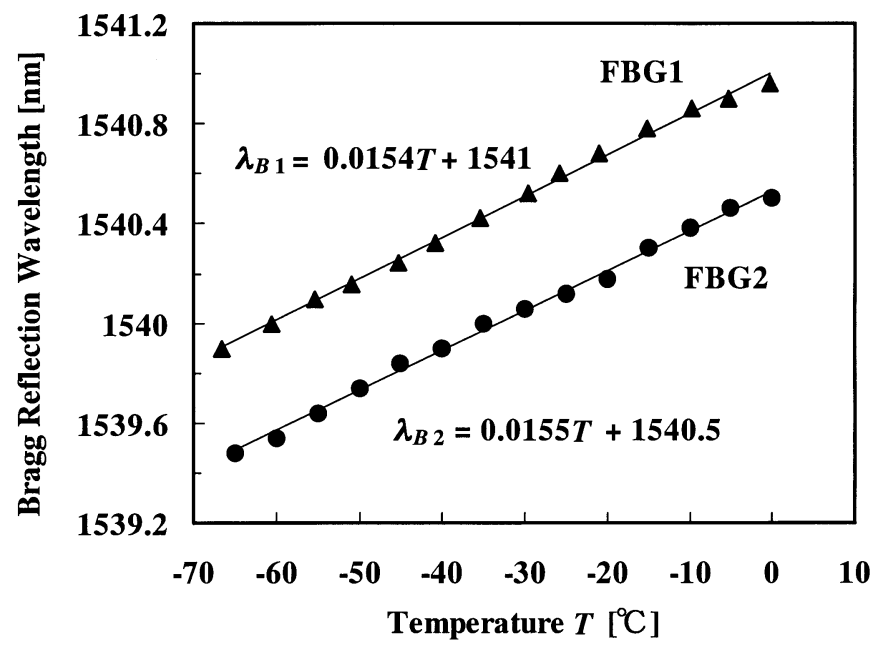

Fig. 4 Temperature dependence of FBG Bragg reflection wavelength. $\lambda_{\mathrm{B} 1}$ and $\lambda_{\mathrm{B} 2}$ are the Bragg reflection wavelengths of FBG1 and FBG2, respectively.

detected with a photodiode. The detected signal is observed on an oscilloscope and measured on an spectrum analyzer. An optical spectrum analyzer is inserted after the circulator 1 to monitor the optical source.

\section{Results and discussion}

Figure 5 shows a typical spectrum of the detected signal. The vibration amplitude is $475 \mathrm{~nm}$ at the end surface of the vibrator, which is calibrated with the optical interference method. The signal-to-noise ratio of the detected signal is about $40 \mathrm{~dB}$ and the second harmonic component in the spectrum is below the noise level in Fig. 5. The sensor operation is then believed to be linear. Figure 6 shows the sensor outputs in arbitrary unit as functions of FBG temperature while keeping the driving voltage and frequency of the vibrator constant. To control the temperature of the FBGs, we place them in a Dewar vessel where liquid nitrogen is put in the bottom. The temperature was varied from -64 to $12^{\circ} \mathrm{C}$. The open circles show the output of the sensor when the sensor is not thermally stabilized. The black triangles show

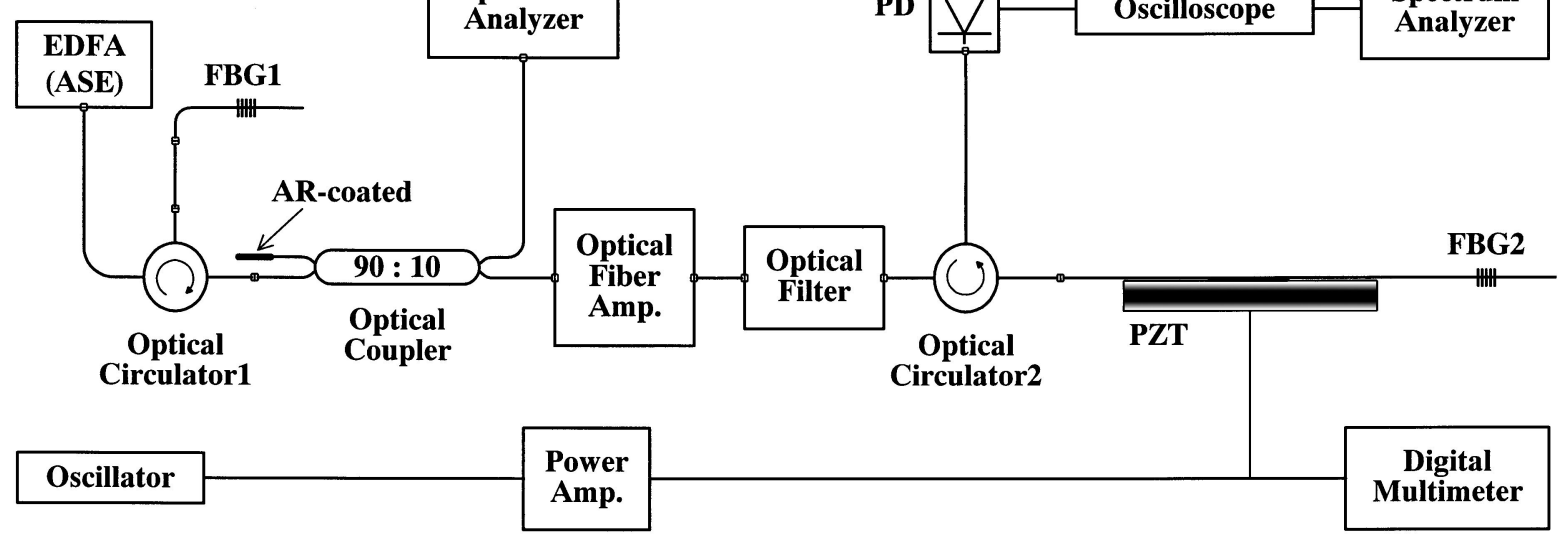

Fig. 3 Experimental setup for thermally stabilized FBG vibration sensor. 


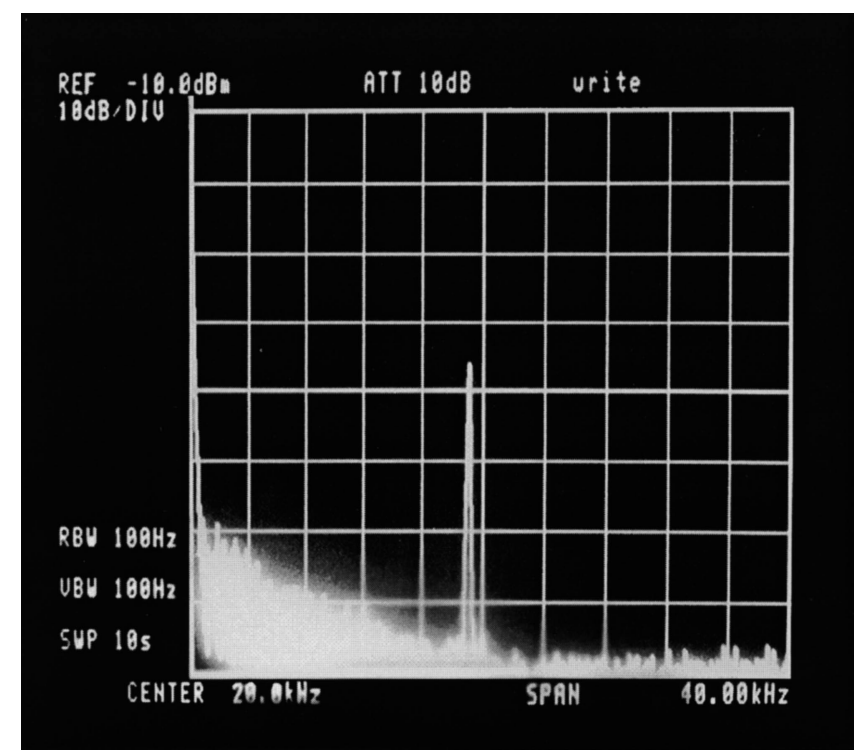

Fig. 5 Spectrum of detected signal.

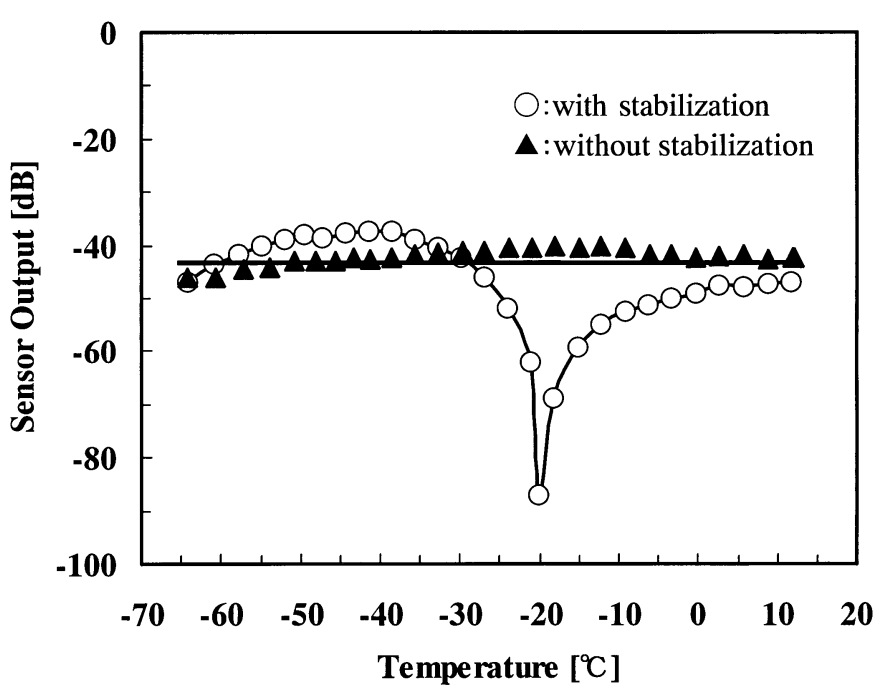

Fig. 6 Temperature dependence of FBG vibration sensor output.

the sensor output when FBG1 is placed in the neighborhood of FBG2 and the temperatures of the two FBGs are varied together, i.e., the sensor is thermally stabilized. One notices large variation in the output of the sensor without stabiliza- tion. The minimum output of the sensor at the level of $-87 \mathrm{~dB}$ corresponds to the noise level and the signal level is thought to be lower than $-87 \mathrm{~dB}$. The output variation of the unstabilized sensor is estimated to be more than $50 \mathrm{~dB}$. When the sensor is thermally stabilized, on the other hand, the output variation of the sensor is as small as $4 \mathrm{~dB}$ over the temperature range shown in the figure. The residual variation in the sensor sensitivity with changing temperature may be due to small, uncontrollable difference in temperature between the two FBGs. The careful and precise arrangement of the FBGs might be effective for further reduction of the temperature instability of the sensor. Another possible improvement can come from optimization in choosing an FBG pair. Small discrepancy in temperature dependence of the Bragg reflection wavelength between the two FBGs could cause some variation in the sensor sensitivity.

\section{Conclusion}

Using two FBGs in an FBG vibration sensor system, one for sensing and other for the optical source in combination with a broad-spectrum light source and optical circulator, we have constructed a new type of an FBG vibration sensor with temperature stability. With the proposed configuration, we obtained the sensor output variation as small as $4 \mathrm{~dB}$ over the temperature range from -64 to $12^{\circ} \mathrm{C}$, which was more than $50 \mathrm{~dB}$ without the stabilization. Further development, including the choice of an FBG pair and spatial arrangement of the FBGs, is expected to provide us with smaller variation in the sensor sensitivity against the environmental temperature change.

\section{References}

[1] N. Takahashi, T. Saeki, K. Tetsumura, S. Takahashi and K. Imamura, "Pressure and temperature dependence of fiber Bragg grating for acoustic sensing," J. Mar. Acoust. Soc. Jpn., 26, 231-238 (1999).

[2] A. D. Kersey, M. A. Davis, H. J. Patrick, M. LeBlanc, K. P. Koo, C. G. Askins, M. A. Putnam and E. J. Friebele, "Fiber grating sensors," J. Lightwave Technol., 15, 1442-1463 (1997).

[3] S. C. Kang, S. Y. Kim, S. B. Lee, S. W. Kwon, S. S. Choi and B. Lee, "Temperature-independent strain sensor system using a tilted fiber bragg grating demodulator," IEEE Photon. Technol. Lett., 10, 1461-1463 (1998).

[4] N. Takahashi, K. Yoshimura and S. Takahashi, "Detection of ultrasonic mechanical vibration of a solid using fiber Bragg grating," Jpn. J. Appl. Phys., 39, 3134-3138 (2000). 